\title{
Effects of constrained dynamic loading, CKIP-1 gene knockout and combination stimulations on bone loss caused by mechanical unloading
}

\author{
BIAO HAN $^{1 *}$, SHU-PING WEI $^{1 *}$, XIN-CHANG ZHANG $^{2}$, HAO LI $^{1}$, \\ YU LI ${ }^{2}$, RUI-XIN LI ${ }^{1}, \mathrm{KAIREN} \mathrm{LI}^{1}$ and XI-ZHENG ZHANG ${ }^{1}$ \\ ${ }^{1}$ Department of Biomedical Engineering and Medical Technology, Tianjin Institute of Medical Equipment, \\ Academy of Military Medical Sciences, Tianjin 300161; ${ }^{2}$ Department of Clinical Medicine, \\ Logistical College of People's Armed Police Forces, Tianjin 300162, P.R. China
}

Received January 12, 2018; Accepted June 20, 2018

DOI: $10.3892 / \mathrm{mmr} .2018 .9222$

\begin{abstract}
Mechanical stimulation plays an important role in maintaining the growth and normal function of the skeletal system. Mechanical unloading occurs, for example, in astronauts spending long periods of time in space or in patients on prolonged bed rest, and causes a rapid loss of bone mass. Casein kinase 2-interacting protein-1 (CKIP-1) is a novel negative bone regulation factor that has been demonstrated to reduce bone loss and enhance bone formation. The aim of this study was to investigate the effect of constrained dynamic loading (Loading) in combination with CKIP-1 gene knockout (KO) on unloading-induced bone loss in tail-suspension mice. The blood serum metabolism index [alkaline phosphatase (ALP) activity and osteocalcin (OCN) levels], tibia mechanical behavior (including bone trabecular microstructure parameters and tibia biomechanical properties), osteoblast-related gene expression [ALP, OCN, collagen I and bone morphogenetic protein-2 and osteoprotegerin (OPG)] and osteoclast-related gene expression [receptor activators of NF-kB ligand (RANKL)] were measured. The results demonstrated that mice experienced a
\end{abstract}

Correspondence to: Professor Xi-Zheng Zhang, Department of Biomedical Engineering and Medical Technology, Tianjin Institute of Medical Equipment, Academy of Military Medical Sciences, 106 Wandong Road, Hedong, Tianjin 300161, P.R. China

E-mail: z84656716@yaoo.com

*Contributed equally

Abbreviations: CKIP-1, casein kinase 2-interacting protein-1; ALP, alkaline phosphatase; OCN, osteocalcin; Col I, collagen I; Smurf1, Smad ubiquitination regulatory factor 1; BMP, bone morphogenetic protein; CK2, casein kinase-2; BMP-2, bone morphogenetic protein-2; PH, pleckstrin homology; RT-qPCR, reverse transcription-quantitative polymerase chain reaction

Key words: mechanical unloading, bone loss, constrained dynamic loading, CKIP-1 knockout, bone formation, bone resorption loss of bone mass after four weeks of tail suspension compared with a wild type group. The mechanical properties, microarchitecture and mRNA expression were significantly increased in mice after Loading $+\mathrm{KO}$ treatment $(\mathrm{P}<0.05)$. Furthermore, compared with loading or $\mathrm{KO}$ alone, the ratio of OPG/RANKL was increased in the combined treatment group. The combined effect of Loading $+\mathrm{KO}$ was greater than that observed with loading or $\mathrm{KO}$ alone $(\mathrm{P}<0.05)$. The present study demonstrates that Loading $+\mathrm{KO}$ can counter unloading-induced bone loss, and combining the two treatments has an additive effect. These results indicate that combined therapy could be a novel strategy for the clinical treatment of disuse osteoporosis associated with space travel or bed rest.

\section{Introduction}

With an increase in the average age of the population, disuse osteoporosis has become a serious health issue in the elderly (International Osteoporosis Foundation, http://www.iofbonehealth.org/). Furthermore, young adults who are bedridden for long periods of time due to conditions such as joint arthrodesis or paralysis may also suffer from unloading-induced loss of bone mass (1). Following long periods in zero gravity, healthy astronauts suffer from a rapid loss of bone mass, which can seriously affect their health and re-adaptation to Earth's gravity (2-4). There is a well-established link between disuse and bone mass loss, and mechanical unloading is known to be responsible for suppressing bone formation and activating bone resorption. The underlying mechanism responsible for disuse-induced bone loss has been investigated in a number of studies (1-6). However, there are few therapies that are able to effectively counteract unloading-induced bone mass loss. Many of the traditional treatments for disuse osteoporosis work by preventing bone resorption rather than promoting bone formation $(7,8)$. It is therefore necessary to find an effective treatment to prevent bone loss and increase bone formation in mechanical unloading-induced disuse osteoporosis.

Mechanical load is critical for maintaining the integrity and normal function of the skeletal system (9). According to Wolff's law and Frost's 'mechanostat', bone growth and bone loss are 
stimulated by the local mechanical elastic deformation of bone, which defines bone modeling and remodeling $(10,11)$. Bone formation varies depending on load amplitude and frequency. Loading frequencies in the range of $10-30 \mathrm{~Hz}$ are optimal for promoting osteogenic activity and bone formation $(12,13)$. Our previous study revealed that constrained dynamic loading can increase bone formation, which in turn increases bone density to counter bone loss caused by ovariectomy (14).

Casein kinase 2-interacting protein-1 (CKIP-1) is considered to be a novel negative bone regulation factor that primarily influences bone formation as opposed to bone resorption $(15,16)$. There is a reciprocal relationship between CKIP-1 and Smad1/5 (16). Smad1/5 are significant signaling molecules in the bone morphogenetic protein (BMP) signaling pathway and play an important role in bone formation. CKIP-1-deficient mice have been reported to have higher bone density, higher bone formation rate and increased ALP activity compared with normal mice. These differences are also exacerbated by age. CKIP-1 knockout in osteoblasts dramatically increases the differentiation and proliferation of osteoblasts and promotes osteogenesis (16). Baas et al reported that CKIP-1 knockout has a significant effect on myoblast fusion in zebra fish (17). A recent study showed that CKIP-1 regulates bone formation in rat mandibular distraction osteogenesis via the Wnt3 $\alpha / \beta$-catenin signaling pathway (18). Furthermore, an across species CKIP-1 siRNA sequence was used in human, rhesus, rat and mouse osteoblast-like cells in vitro to promote osteogenic differentiation without any immunostimulatory activity, and was demonstrated to increase bone formation and attenuate bone loss in osteoporotic mice (19). A previous study reported that NT-GPP/siCKIP-1 promotes osteogenic differentiation in MC3T3-E1 cells, increases the expression of ALP and Col-I and enhances extracellular matrix (ECM) mineralization to increase bone formation in vivo (20). These results indicate that CKIP-1 may be a potential target for the clinical treatment of osteoporosis.

Mechanical loading and CKIP-1 gene knockout have been demonstrated to play important roles in the regulation of bone remodeling. However, to the best of our knowledge, the combination of mechanical strain and CKIP-1 gene knockout has not yet been investigated. The National Aeronautics and Space Administration (NASA) reported that the combination of exercise and medicine (alendronate pill and vitamin D) was more effective in preventing bone and muscle mass loss in astronauts compared to treatment using exercise or medication alone (21). Our previous study also demonstrated that the combination of mechanical strain stimulation and icariin improved osteoinduction in MC3T3-E1 cells and ovariectomy-induced osteoporotic mice $(22,23)$.

The objective of this study was to explore the effect of constrained dynamic loading combined with CKIP-1 gene knockout on bone loss in tail-suspension mice. Markers of bone formation and resorption were assessed. The results of the present study may provide a basis for a novel treatment protocol for disuse osteoporosis.

\section{Materials and methods}

Animals and reagents. Wild type (WT) C57BL/6J mice were purchased from the Military Academy of Medical Science
(Beijing, China). CKIP-1-deficient mice were donated by Professor Ling-qiang Zhang (Beijing Proteomics Research Center, Beijing, China). CKIP-1-deficient and WT C57BL/6J mice were backcrossed more than 10 times and the genotype of the offspring was determined using PCR. All mice were housed in the animal room of our research laboratory. The present study was conducted according to the Guide for the Care and Use of Laboratory Animals published by the US National Institutes of Health (NIH Publication 8th edition, 2011). The animal experimental protocol was approved by the Academy of Military Medical Sciences (Beijing, China). A mouse OCN ELISA kit was purchased from Cloud-Clone Corp. (Houston, TX, USA). A tail DNA determination kit and an ALP assay kit were obtained from the Nanjing Jiancheng Bioengineering Institute (Nanjing, China). SYBR Green MIX and TRIzol were purchased from Thermo Fisher Scientific, Inc., Waltham, MA, USA). High-capacity cDNA reverse transcription kits were purchased from Thermo Fisher Scientific, Inc.

Experiment design. A total of 48 WT C57BL/6J mice (2-3 months old) were randomly separated into three groups: WT (untreated wild type mice; $n=16$ ), TS (mice underwent tail-suspension; $\mathrm{n}=16$ ) and TS + Loading (mice underwent tail-suspension and constrained dynamic loading; $n=16$ ). A total of 32 CKIP-1 knockout were separated into two groups: TS + KO (underwent tail suspension; $\mathrm{n}=16$ ) and $\mathrm{TS}+$ Loading $+\mathrm{KO}$ (underwent tail suspension and constrained dynamic loading; $n=16)$. For tail suspension, mice were allowed to acclimate for one week and tail suspension was carried out as previously described $(24,25)$. Mice in the TS + Loading and TS + Loading + KO groups underwent constrained dynamic loading on their left tibia (the peak strain of the middle tibia was $\sim 2,500 \mu \varepsilon$ ) Mice were subsequently sacrificed and the tibias were removed without soft tissue. Serum was also collected.

Operation of constrained dynamic loading. In our previous study, using FEA (Finite Element Analysis), curve-fitting and theoretical calculations on mouse tibia bone model, we finally choose a sinusoidal displacement curve $(\mathrm{y}=0.045 \mathrm{sin}$ $(30 \pi \times \mathrm{t})(\mathrm{mm}))$. With $0.5 \mathrm{~N}$ preload and $0.045 \mathrm{~mm}$ amplitude, the diaphyseal peak strain on the tibia will be in a range of 220 and 3,000 $\mu \varepsilon$. However, consider the real situation of bone tissue, the actual diaphyseal peak strain on the tibia will be in 1,000 and 3,000 $\mu \varepsilon$ range (the vast majority was 2,500 $\mu \varepsilon$ ) after the impact energy was absorbed by the joint and muscle. Then, we use the Instron 5685 (Instron, Norwood, MA, USA) to perform constrained dynamic loading on their left tibia with a frequency of $15 \mathrm{~Hz}$ for 30 min every 3 days over a period of 4 weeks (14).

Mechanical behaviour of tibias. Micro CT images (Skyscan 1076; SkyScan, Kontich, Belgium) were used to analyse bone microarchitecture. The scanning voltage was set at $50 \mathrm{KV}$, and the scanning resolution was $9 \mu \mathrm{m}$. A 3-mm proximal area of cancellous bone was selected as the region of interest. The bone volume fraction (BV/TV), trabecular number (Tb.N), trabecular thickness (Tb.Th), trabecular pattern factor (Tb.Pf) and trabecular separation (Tb.Sp) were measured. The classical three-point bending test was conducted using an Instron 5685 (Instron) in order to determine the tibial biomechanical 
Table I. Primers sequences in this study.

\begin{tabular}{lll}
\hline Gene & \multicolumn{1}{c}{ Forward: 5'-3' } & \multicolumn{1}{c}{ Reverse: $5^{\prime}-3^{\prime}$} \\
\hline BMP-2 & TGTGAGGATTAGCAGGTCTT & TCGTTTGTGGAGCGGATG \\
COL-I & CAGTGGCGGTTATGACTT & CTGCGGATGTTCTCAATCT \\
ALP & AAGGAGGCAGGATTGAC & ATCAGCAGTAACCACAGTC \\
OCN & CCATCTTTCTGCTCACTCT & ACTACCTTATTGCCCTCCT \\
OPG & AGTCTGAGGAAGACCATGAG & AAACAGCCCAGTGACCATTC \\
RANKL & CCAAGATGGCTTCTATTACC & TCCCTCCTTTCATCAGGTTAT \\
$\beta$-actin & GATTACTGCTCTGGCTCCT & ACTCCTGCTTGCTGATCC
\end{tabular}

BMP-2, bone morphogenetic protein 2; COL-I, collagen type I; ALP, alkaline phosphatase; OCN, osteocalcin; OPG, osteoprotegerin; RANKL, receptor activators of $\mathrm{NF}-\kappa \mathrm{B}$ ligand.

properties. $0.1-\mathrm{N}$ preloading force and a $3-\mathrm{mm} / \mathrm{min}$ loading rate were applied, and fracture load, fracture energy, fracture displacement and fracture stress were measured.

Bone turnover marker evaluation. Serum was separated by centrifugation at $1,000 \mathrm{x}$ g for $15 \mathrm{~min}$. Serum alkaline phosphatase (ALP) activity and osteocalcin (OCN) content were determined using the ALP kit and OCN ELISA kit, respectively, according to the manufacturer's instructions.

Real-time PCR analysis. A liquid nitrogen grinding method was applied on the collected tibias. Total RNA was extracted using TRIzol according to the manufacturer's instructions. cDNA was synthesized using a high-capacity cDNA reverse transcription kit. The primer sequences are listed in Table I. PCR reactions were performed in $25-\mu 1$ reaction volumes with $12.5 \mu \mathrm{l}$ SYBR Green PCR master mix and $1 \mu 1$ each primers. Thermocycling conditions were as follows: $95^{\circ} \mathrm{C}$ for $2 \mathrm{~min}$ followed by 38 cycles of $95^{\circ} \mathrm{C}$ for $15 \mathrm{sec}, 60^{\circ} \mathrm{C}$ for $30 \mathrm{sec}$ and melting at $55-95^{\circ} \mathrm{C}$ for $30 \mathrm{sec}$ per $0.5^{\circ} \mathrm{C}$. Samples were analyzed using the $2^{-\Delta \Delta \mathrm{Cq}}$ method (26). The results were calculated relative to the TS group after normalization to the $\beta$-actin gene.

Data analysis. Data were expressed as the mean \pm SD from at least three independent experiments. One-way analysis of variance (ANOVA) was used to analyze the experimental data. Tukey's post hoc test was used to compare the groups. $\mathrm{P}<0.05$ indicated that results were significantly different.

\section{Results}

Expression of CKIP-1 in unloaded bone in WT mice. We first determined changes in the expression of CKIP-1 in the bone after tail suspension for $0,1,2,3$ or 4 weeks. As shown in Fig. 1, the expression of CKIP-1 mRNA was decreased at week 1 ( $\mathrm{P}>0.05)$; however, no significant differences in CKIP-1 expression were observed at week 3 or 4 compared with week 2 .

Effects of loading + KO on trabecular microarchitecture. We examined the effects of combined treatment with constrained dynamic loading and CKIP-1 gene knockout after 4 weeks of tail-suspension. As shown in Fig. 2, micro-CT and histomorphometric analysis of the trabecular bone showed that

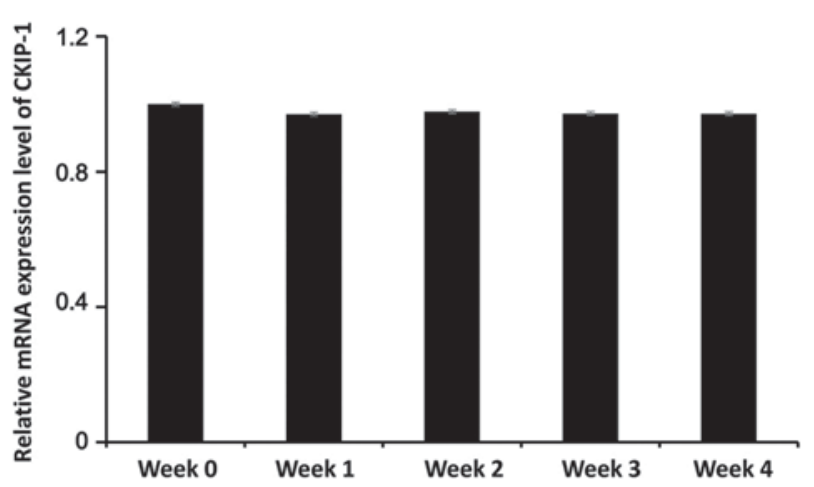

Figure 1. CKIP-1 expression in mouse tibias following tail suspension for 0 , $1,2,3$ and 4 weeks. Results are shown as the mean \pm SD from three independent experiments. CKIP-1, casein kinase 2-interacting protein-1.

TS significantly reduced the relative bone volume (BV/TV), trabecular thickness (Tb.Th) and trabecular number (Tb.N) compared with WT mice. In contrast, trabecular separation (Tb.Sp) and trabecular pattern factor (Tb.Pf) were significantly increased compared with WT mice. After treatment with Loading, KO, or Loading + KO, trabecular microarchitecture was improved, with significant increases in relative bone volume (BV/TV), trabecular thickness (Tb.Th) and trabecular number (Tb.N), as well as reduced trabecular separation (Tb.Sp) and trabecular pattern factor (Tb.Pf) relative to the TS group $(\mathrm{P}<0.05)$. Compared to treatment with Loading or $\mathrm{KO}$ alone, combined Loading + KO treatment resulted in a significantly greater improvement in trabecular microarchitecture in mouse tibias $(\mathrm{P}<0.05)$.

Effects of Loading + KO on tibia mechanical properties. The macro-mechanical properties of tibia bone tissues are shown in Fig. 3. Fracture load, fracture energy, fracture displacement and fracture stress were significantly reduced $(\mathrm{P}<0.05)$ in the TS group after 4 weeks of tail suspension compared with the WT group. The biomechanical properties were significantly $(\mathrm{P}<0.05)$ improved in the Loading, $\mathrm{KO}$ and Loading $+\mathrm{KO}$ groups compared with the TS group. Consistent with the results for trabecular microarchitecture, mechanical properties in the Loading + KO group were significantly improved compared with the Loading and KO groups $(\mathrm{P}<0.05)$. 
A
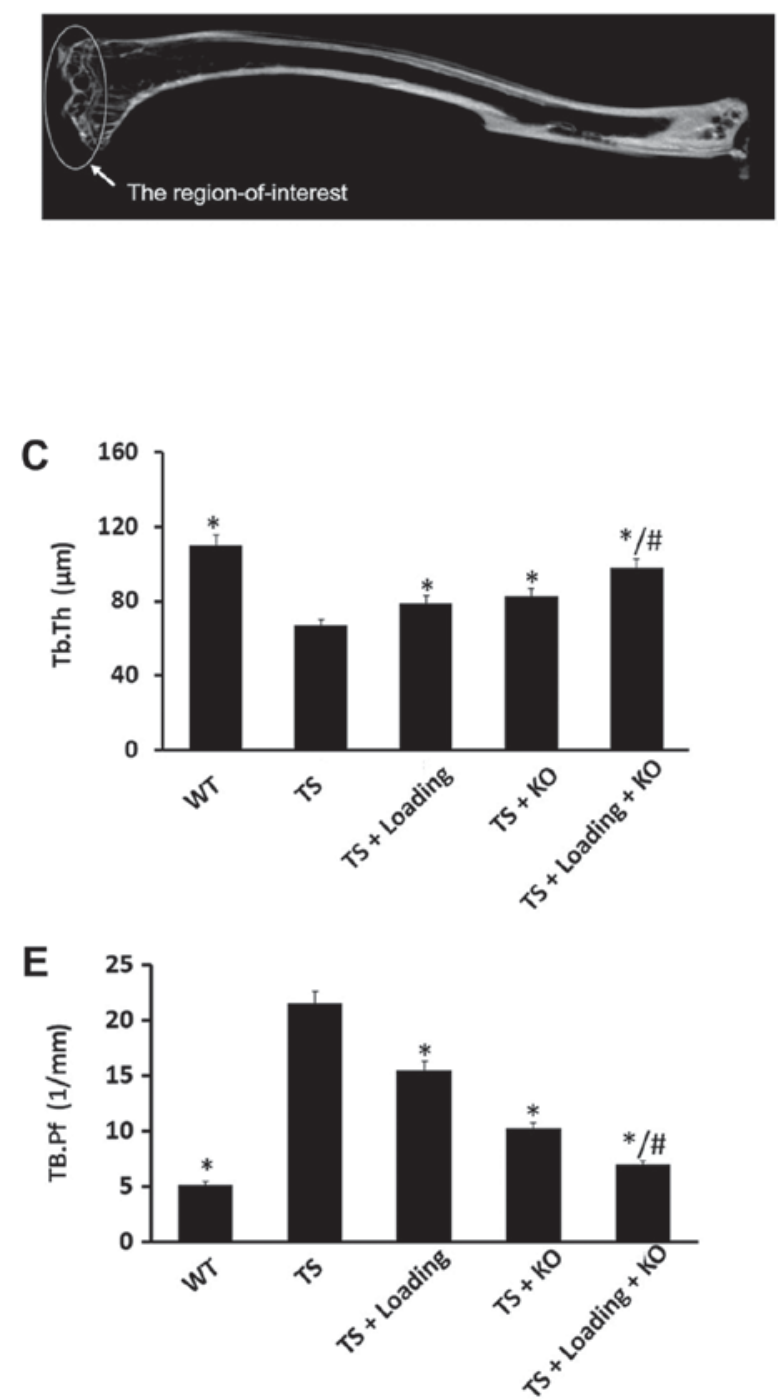
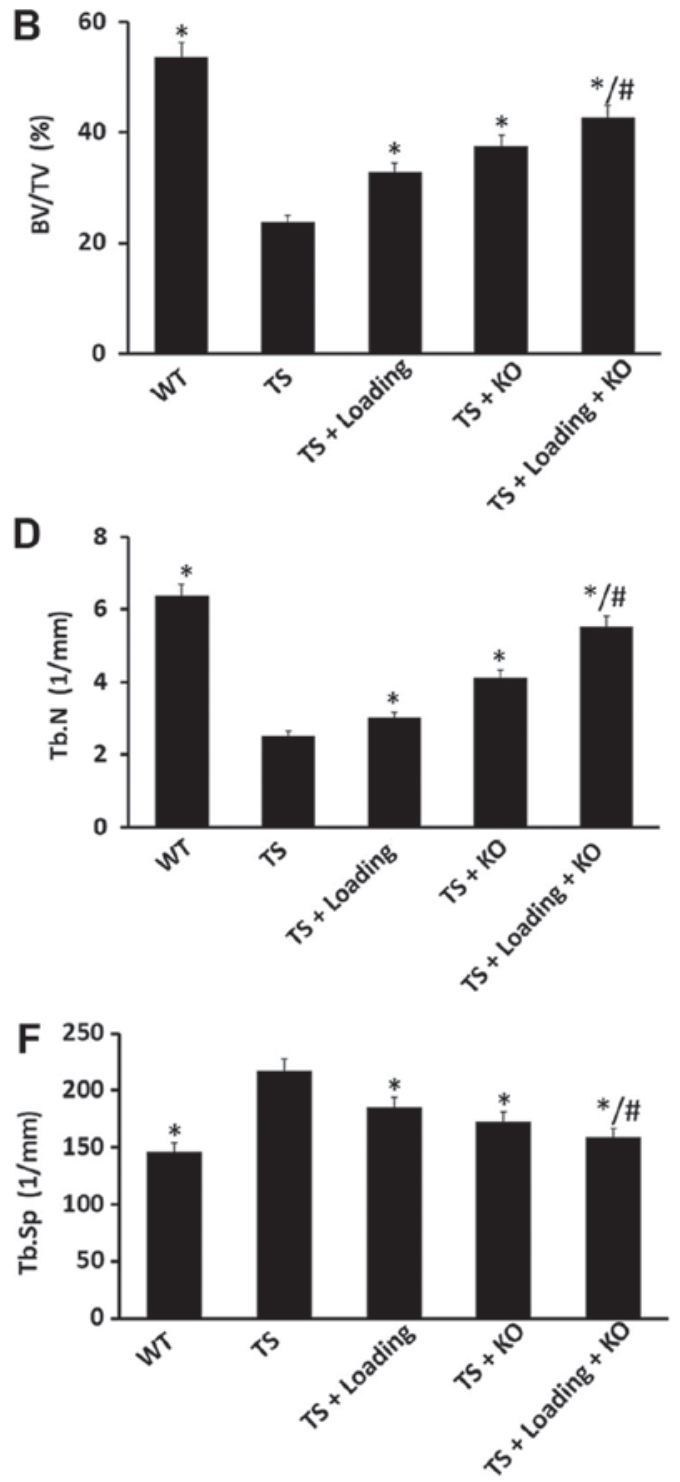

Figure 2. Effects of Loading, KO and Loading + KO on tibia cancellous bone microarchitecture as measured by micro-CT. (A) Tibia bone region-of-interest, (B) BV/TV, (C) Tb.Th, (D) Tb.N, (E) Tb.Pf and (F) Tb.Sp in different groups. Results are shown as the mean \pm SD from three independent experiments. ${ }^{*} \mathrm{P}<0.05$ vs. WT, ${ }^{* *} \mathrm{P}<0.05$ vs. TS, ${ }^{\text {}} \mathrm{P}<0.05$ vs. TS + Loading or TS + KO. WT, wild type; TS, tail suspension; Loading, constrained dynamic loading; KO, CKIP-1 gene knockout; BV/TV, bone volume fraction; Tb.N, trabecular number; Tb.Th, trabecular thickness; Tb.Pf, trabecular pattern factor; Tb.Sp, trabecular separation.

Effect of Loading + KO on ALP activity and OCN content in serum. ALP and OCN are markers of osteoblast differentiation and osteogenic activity. As shown in Fig. 4, the serum ALP activity and OCN content were significantly lower $(\mathrm{P}<0.05)$ following 4 weeks of tail suspension compared with the WT group. However, ALP activity and OCN content were significantly higher $(\mathrm{P}<0.05)$ in the Loading, $\mathrm{KO}$ and Loading + KO groups compared with the TS group. Moreover, compared with the Loading group, serum ALP activity and OCN content were significantly higher in the Loading + KO group $(\mathrm{P}<0.05)$. However, no significant difference in ALP activity or OCN content was observed between the KO and Loading + KO groups.

Effect of loading + KO on BMP-2, Col-I, ALP, and OCN $m R N A$ expression. The expression of BMP-2, Col-I, ALP and OCN mRNA was investigated using RT-qPCR. As shown in Fig. 5, the expression of BMP-2, Col-I, ALP and OCN mRNA was significantly downregulated in the group treated with 4 weeks of tail suspension $(\mathrm{P}<0.05)$ compared with the WT group. However, compared with the TS group, the expression of BMP-2, Col-I, ALP and OCN mRNA was significantly upregulated $(\mathrm{P}<0.05)$ in the Loading, $\mathrm{KO}$ and Loading $+\mathrm{KO}$ groups. Moreover, treatment with Loading $+\mathrm{KO}$ resulted in a significantly greater upregulation of BMP-2, Col-I, ALP and OCN expression $(\mathrm{P}<0.05)$ compared with $\mathrm{KO}$ alone.

Effect of Loading $+K O$ on $O P G / R A N K L$ ratio. The $\mathrm{OPG} / \mathrm{RANKL}$ ratio is a critical factor in the regulation of bone metabolism, especially in osteoblast and osteoclast development. As shown in Fig. 6, the expression of OPG mRNA in the TS group was significantly decreased $(42.65 \%$; $\mathrm{P}<0.05)$ compared with the WT group, while RANKL expression was upregulated $(12.24 \%$; P $>0.05)$. Compared with the TS group, RANKL expression was downregulated in the 

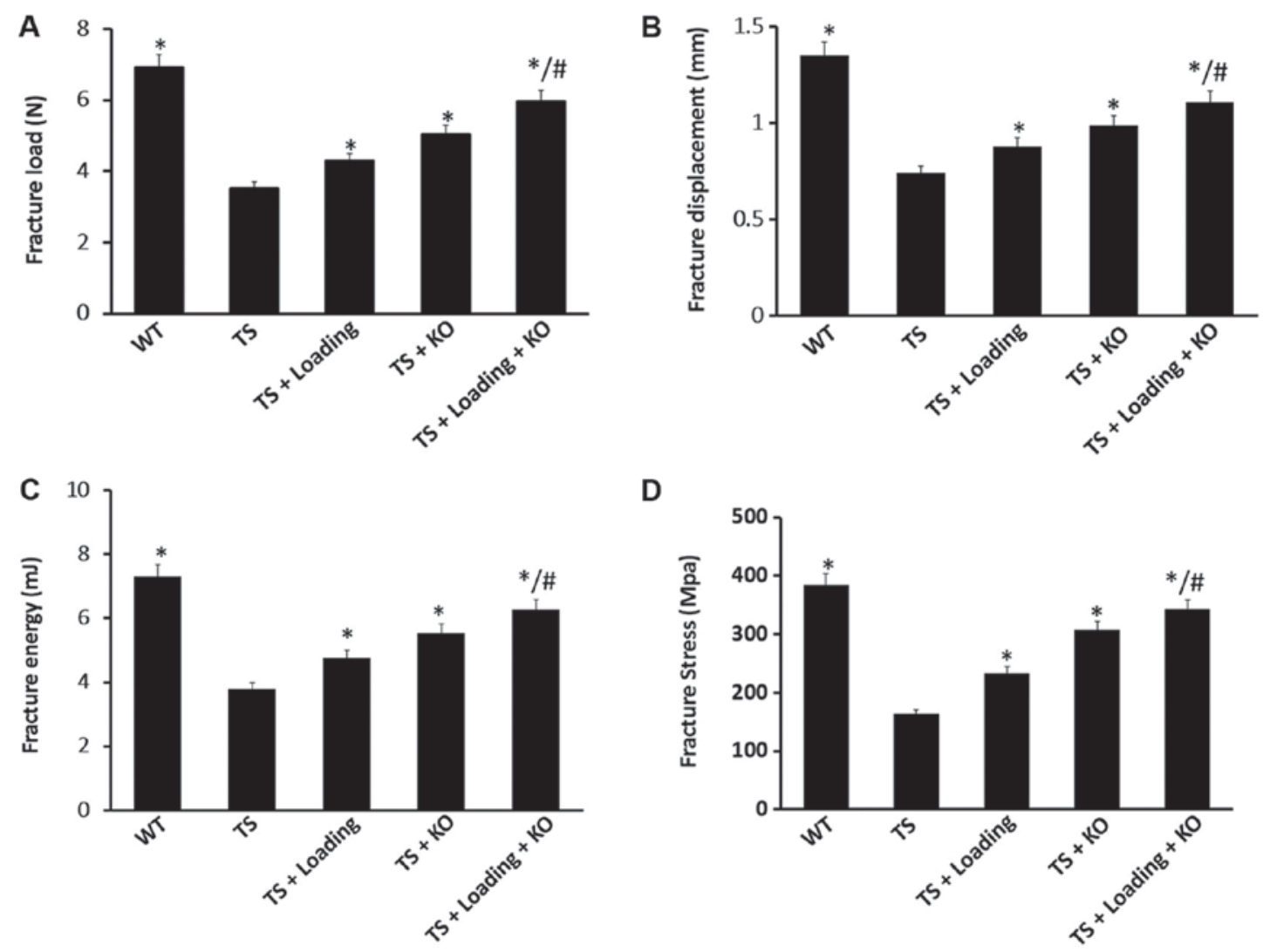

Figure 3. Effects of Loading, $\mathrm{KO}$ and Loading $+\mathrm{KO}$ on tibia cancellous bone biomechanical properties as assessed using the three-point bending test. (A) Fracture load, (B) fracture energy, (C) fracture displacement and (D) fracture stress in different groups. Results are shown as the mean \pm SD from three independent experiments. "P $<0.05$ vs. WT, ${ }^{* *} \mathrm{P}<0.05$ vs. TS, ${ }^{*} \mathrm{P}<0.05$ vs. TS + Loading or TS + KO. WT, wild type; TS, tail suspension; Loading, constrained dynamic loading; KO, CKIP-1 gene knockout.

Loading (12.13\%; P>0.05), KO (12.01\%; P>0.05) and Loading $+\mathrm{KO}(33.34 \% ; \mathrm{P}<0.05)$ groups, while OPG expression was upregulated $(19.39 \%, \mathrm{P}<0.05 ; 20.94 \%, \mathrm{P}<0.05 ; 50.01 \%, \mathrm{P}<0.05$, respectively). The OPG/RANKL ratio was increased following treatment with Loading, $\mathrm{KO}$ and Loading $+\mathrm{KO}$. Furthermore, compared with Loading or $\mathrm{KO}$ alone, Loading $+\mathrm{KO}$ resulted in a greater increase in the $\mathrm{OPG} / \mathrm{RANKL}$ ratio.

\section{Discussion}

Mechanical unloading had serious harmful effects on the cardiovascular system, the brain and bone metabolism. A loss of mechanical stress may lead to bone loss and disuse osteoporosis. For example, one month of microgravity may result in a loss of bone mass equivalent to that experienced by a postmenopausal woman in one year (27-29). Drug treatment has limitations, including inhibited bone resorption and no increase in bone formation, while physical therapy takes a long time to take effect. As such, combined treatment with gene therapy and physical methods may be a better choice. CKIP-1 is a scaffold protein with excellent osteogenic effects and no immunostimulatory effects (19). Our previous study revealed that constrained dynamic loading could strengthen bone structure and reduce bone fractures. Apparent strain $(1,200 \mu \varepsilon, 2,500-2,800 \mu \varepsilon)$ can improve osteoblast proliferation and differentiation, increase osteoblast activity and counter osteoporosis in ovariectomized mice in vitro and
MC3T3-E1 cells in vivo (30-32). In the present study, we first explored the effect of constrained dynamic loading in combination with CKIP-1 knockout in a tail suspension mouse model. The results show that unloading due to tail suspension damaged the trabecular microarchitecture, downregulated osteogenic-related gene expression, reduced tibial mid-shaft strength properties and decreased the OPG/RANKL ratio. These results demonstrate the process of bone loss due to disuse osteoporosis in healthy young mice. Osteoporosis is a condition characterized by progressive loss of bone density, thinning of bone tissue and increased vulnerability to fractures. We found that constrained dynamic loading and CKIP-1 knockout can counter unloading-induced bone loss or disuse osteoporosis, promote bone formation, enhance the bone strength and reduce fracture risk, and the combination of both treatments significantly increases this effect. These results indicate that combined therapy with constrained dynamic loading and CKIP-1 knockout may be an effective treatment for disuse-induced bone loss or osteoporosis in mice.

To confirm whether unloading has an effect on CKIP-1, we measured the level of CKIP-1 mRNA in mice following tail suspension. Compared to week 0, CKIP-1 mRNA was downregulated at other time points; however, no significant difference was observed. In our previous study, gene chip and bioinformatics technology was used, and we found that CKIP-1 was the target gene of miR-98-5p in MC3T3-E1 cells (33). In addition, miR-98-5p was found to be upregulated 

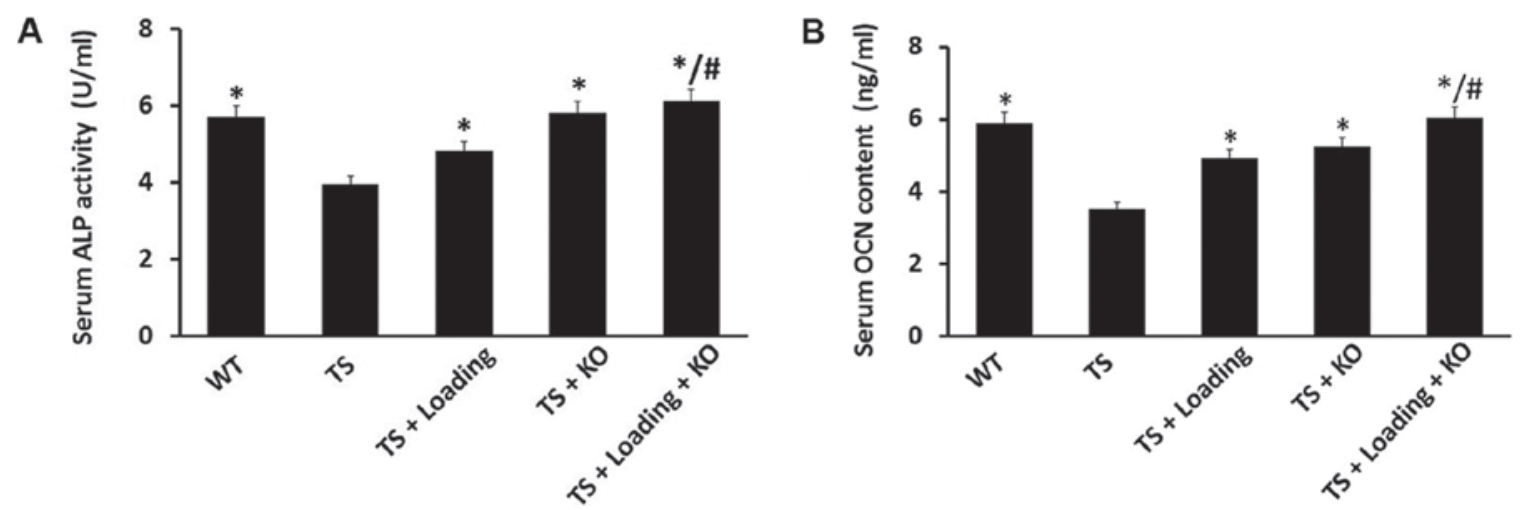

Figure 4. Serum (A) ALP activity and (B) OCN content were measured using ELISA kits. Results are shown as the mean \pm SD from three independent experiments. ${ }^{*} \mathrm{P}<0.05$ vs. WT, ${ }^{* *} \mathrm{P}<0.05$ vs. TS, ${ }^{*} \mathrm{P}<0.05$ vs. TS + Loading or TS + KO. WT, wild type; TS, tail suspension; Loading, constrained dynamic loading; KO, CKIP-1 gene knockout; ALP, alkaline phosphatase; OCN, osteocalcin.
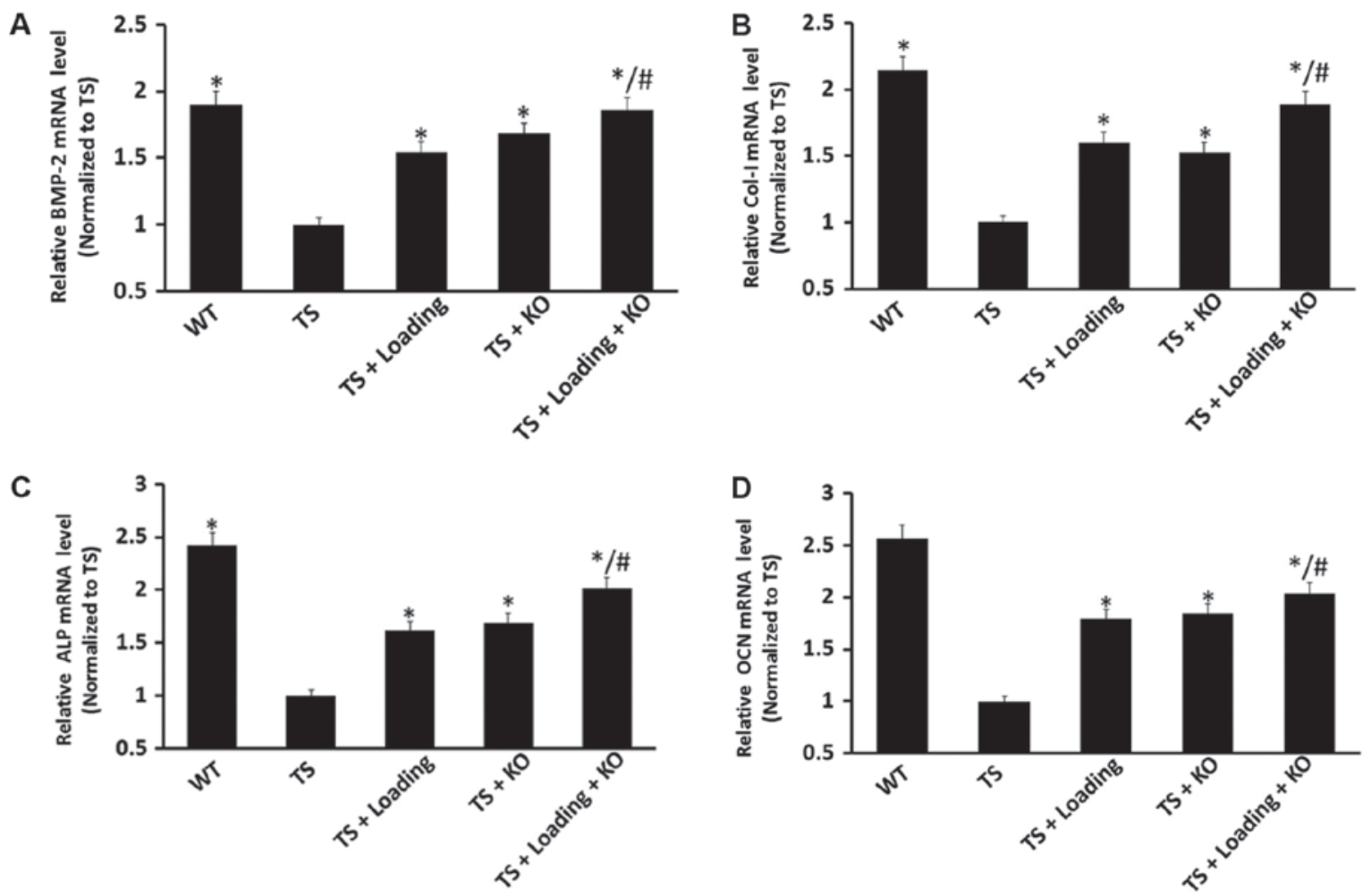

Figure 5. Effect of Loading, $\mathrm{KO}$ and Loading $+\mathrm{KO}$ on the expression of osteoblast differentiation markers following tail suspension. Expression of (A) BMP-2, (B) COL-I, (C) ALP and (D) OCN mRNA as assessed by qRT-PCR. Results are shown as the mean \pm SD from three independent experiments. "P $<0.05$ vs. WT, ${ }^{* *} \mathrm{P}<0.05$ vs. TS, ${ }^{\text {"P }}<0.05$ vs. TS + Loading or TS + KO. WT, wild type; TS, tail suspension; Loading, constrained dynamic loading; KO, CKIP-1 gene knockout; BMP-2, bone morphogenetic protein-2; Col I, collagen I; ALP, alkaline phosphatase; OCN, osteocalcin; qRT-PCR, reverse transcription-quantitative polymerase chain reaction.

under mechanical unloading conditions (data unpublished). It was therefore hypothesized that the downregulation of CKIP-1 mRNA following tail suspension may be associated with an accumulation of miR-98-5p.

Bone microarchitecture and biomechanical properties are important indicators used to evaluate, diagnose and treat bone loss or osteoporosis $(34,35)$. Micro-CT is a conventional method that can be used to directly observe changes in bone microarchitecture. The classical three-point bending test is a method used to measure bone biomechanical properties in macromechanics. Osteoporosis is characterized by bone loss and an increased risk of bone fractures. In the present study, mice tibial metaphyseal bone structure and function was significantly decreased after four weeks of tail suspension, mainly by reducing the bone volume fraction, trabecular number and trabecular thickness, and increasing the trabecular pattern factor and trabecular separation, which is consistent with the results of previous studies $(36,37)$. The three-point bending test results revealed lower fracture load, fracture energy, fracture displacement and fracture stress, in accordance with our previous study (30). These results confirmed that the disuse osteoporosis model had been successfully constructed and that 

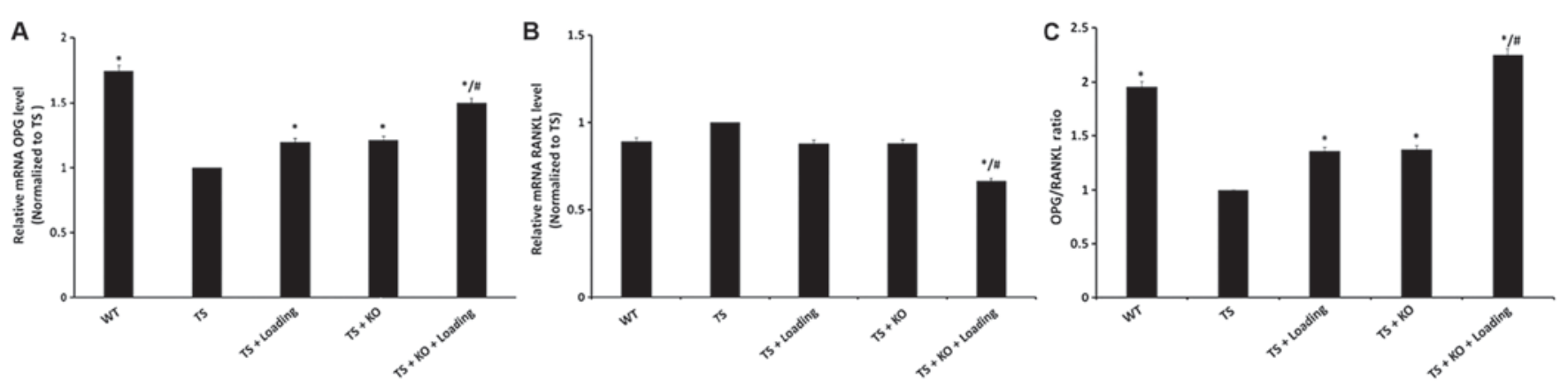

Figure 6. Effect of Loading, KO and Loading + KO on the expression of (A) OPG and (B) RANKL mRNA and the (C) OPG/RANKL ratio. Results are shown as the mean $\pm \mathrm{SD}$ from three independent experiments. ${ }^{*} \mathrm{P}<0.05$ vs. WT, ${ }^{* *} \mathrm{P}<0.05$ vs. TS, ${ }^{*} \mathrm{P}<0.05$ vs. TS + Loading or TS + KO. WT, wild type; TS, tail suspension; Loading, constrained dynamic loading; KO, CKIP-1 gene knockout; OPG, osteoprotegerin; RANKL, receptor activators of NF- $\mathrm{kB}$ ligand.

mechanical unloading is a critical factor in bone loss. One of the aims of the present study was to compare the effects of Loading, $\mathrm{KO}$ and Loading $+\mathrm{KO}$ on bone loss in mice who had been subjected to unloading. In this study, treatment with Loading, $\mathrm{KO}$ or Loading + KO effectively maintained bone mass, suppressed the destruction of bone microarchitecture and increased bone formation following tail suspension. Loading and $\mathrm{KO}$ treatments were demonstrated to prevent bone loss caused by unloading and there was an additive effect of Loading + KO.

As well as analyzing bone microarchitecture and bone biomechanical properties, we investigated changes in bone-related gene expression. Bone morphogenetic proteins (BMPs) have been shown to play an important role in regulating osteoblast differentiation and bone formation (38). BMP-2 is a critical factor in bone remodeling and rebuilding in the BMP family and is applied in many fields, including tissue engineering, oncology and osteoporosis treatment (39-42). ALP, OCN and Col-I are major biological markers and potent osteoblastic factors (43). Our study showed that the expression of BMP-2 mRNA was significantly increased by dynamic loading or CKIP-1 gene knockout following tail suspension, and that combined treatment with dynamic loading and CKIP-1 gene knockout had a coupling effect. The combination of dynamic loading and CKIP-1 knockout may increase bone formation via the BMP signaling pathway. Furthermore, serum ALP activity, OCN content and the expression of ALP, OCN and Col-I mRNA were consistent with that of BMP-2. The results suggest that combined treatment with dynamic loading and gene knockout (CKIP-1) is more effective than either treatment alone.

The OPG/RANKL pathway affects osteoblasts and osteoclasts and serves an important role in maintaining bone balance. By interacting with the receptor activator of NF- $\kappa \mathrm{B}$ (RANK), RANKL induces osteoclast differentiation and promotes bone resorption. OPG is a decoy receptor and RANKL inhibitor. The OPG/RANKL ratio can be used to measure bone mass loss and bone rebuilding (44-47). The present study showed that Loading $+\mathrm{KO}$ could upregulate the expression of OPG mRNA and downregulate the expression of RANKL mRNA by increasing the OPG/RANKL ratio, suggesting that Loading $+\mathrm{KO}$ not only promotes bone formation but suppresses bone resorption. These results are in agreement with our previous study and indicate a potential mechanism by which Loading $+\mathrm{KO}$ affects bone metabolism.

In our previous study, we found that mechanical loading had no effect on CKIP-1 expression (unpublished). However, previous research has shown that mechanical loading inhibits the expression of Smurf1 while CKIP-1 knockout inhibits Smurf1 activity $(16,48)$. Research indicates that constrained dynamic loading affects osteoblast proliferation and differentiation via the $\mathrm{Wnt} / \beta$-catenin pathway $(49,50)$; moreover, CK2 may bind with phosphorylate signaling molecules in the Wnt signaling pathway to affect gene regulation $(51,52)$. In future studies, we plan to investigate whether CKIP-1 affects the Wnt signaling pathway to regulate bone formation.

In the present study, we assessed biomarkers and analysed bone mechanical behaviour in the early stages of disuse osteoporosis. In future, the long-term effects and treatment should be considered. The present study was limited, as only one species model was utilised; ideally, two or more different species models should be used and immunity problems should be considered.

As an animal study, we first must consider if the present study results can be used for human therapies. Mechanical stimulation (physical exercise) and periodic vibration with high frequency $(10-50 \mathrm{~Hz})$ could reverse bone loss in astronaut, which has been supported by NASA funded doctors $(21,53,54)$. CKIP-1 was widely expressed in musculoskeletal tissues and slightly expressed in liver, kidney and other tissue. In addition, there were no disfunctions in other organs after the CKIP-1 gene knockout in the mice and without any immunostimulatory activity in human monocytes (55). Guo et al reported that using cross-species CKIP-1 siRNA sequence (bone-targeting siRNA, tiny-level exposure in non-skeletal tissues) could increase osteoblast differentiation and stimulated bone formation in vitro and in vivo (19), which suggest us a clinical application for disuse osteoporosis. Taken together, it indicated that the combined therapy may be a potential clinical implication in osteoporosis. However, as a basic research, there is a long way to go to prove whether it can be used in the clinical trial and we believed that the combination stimulations would bring goodly foreground in treating related disease in orthopedics.

In conclusion, we found that constrained dynamic loading and CKIP-1 knockout are effective treatments for mechanical unloading-induced bone loss, and the effect of combined 
treatment is significantly greater compared with either treatment alone. Combined treatment may represent an effective novel treatment for bone injury and rebuilding.

\section{Acknowledgements}

Not applicable.

\section{Funding}

The present study was supported by the National Natural Science Foundation of China (grant no. 31370942 and 11432016).

\section{Availability of data and materials}

The datasets used and/or analyzed during the current study are available from the corresponding author on reasonable request.

\section{Authors' contributions}

$\mathrm{BH}$ conceived and designed the research and drafted the manuscript. SPW and BH acquired data, analyzed and interpreted data, and performed statistical analysis. XCZ, HL, YL, RXL and KL contributed reagents, materials and performed the animal experiments. XZZ conceived and designed the research, and revised the manuscript for important intellectual content. All authors read and approved the final manuscript.

\section{Ethics approval and consent to participate}

The animal experimental protocol was approved by the Academy of Military Medical Sciences (Beijing, China).

\section{Patient consent for publication}

Not applicable.

\section{Competing interests}

The authors declare that they have no competing interests.

\section{References}

1. Jaul E, Malcov T and Menczel J: Osteoporosis in tube-fed bed-ridden elderly female patients. J Am Geriatr Soc 57: 1318-1320, 2009.

2. vico L, Collet P, Guiqnandon A, Lafaqe-Proust MH, Thomas T, Rehaillia $\mathrm{M}$ and Alexandre $\mathrm{C}$ : Effects of long-term microgravity exposure on cancellous and cortical weight-bearing bones of cosmonauts. Lancet 355: 1607-1611, 2000.

3. Landis WJ, Hodgens KJ, Block D, Toma CD and Gerstenfeld LC: Spaceflight effects oncultured embryonic chick bone cells. J Bone Miner Res 15: 1099-1112, 2000.

4. Tavella S, Ruggiu A, Giuliani A, Brun F, Canciani B, Manescu A, Marozzi K, Cilli M, Costa D, Liu Y, et al: Bone turnover in wild type and pleiotrophin-transgenicmice housed for three months in the International Space Station (ISS). PLoS One 7: e33179, 2012.

5. Manolagas SC: Birth and death of bone cells: Basic regulatory mechanisms and implications for the pathogenesis and treatment of osteoporosis. Endocr Rev 21: 115-137, 2000.

6. Judex S, Gupta S and Rubin C: Regulation of mechanical signals in bone. Orthod Craniofac Res 12: 94-104, 2009.

7. Khosla S, Westendorf JJ and Oursler MJ: Building bone to reverse osteoporosis and repair fractures. J Clin Invest 118: 421-428, 2008
8. Rodan GA and Martin TJ: Therapeutic approaches to bone diseases. Science 289: 1508-1514, 2000.

9. Hillam RA and Skerry TM: Inhibition of bone resorptionand stimulation of formation by mechanical loading of the modeling rat ulna in vivo. J Bone Miner Res 10: 683-689, 1995.

10. Frost HM: Bone 'mass' and the 'mechanostat': A proposal. Anat Rec 219: 1-9. 1987.

11. Frost HM: Defining osteopenias and osteoporoses: Another view (with insights from a new paradigm). Bone 20: 385-391, 1997.

12. Robling AG, Hinant FM, Burr DB and Turner CH: Improved bone structure and strength after long-term mechanical loading is greatest if loading is separated into short bouts. J Bone Miner Res 17: 1545-1554, 2002.

13. Kaspar D, Seidl W, Neidlinger-Wilke C, Beck A, Claes L and Ignatius A: Proliferation of human-derived osteoblast-like cells depends on the cycle number and frequency of uniaxial strain. J Biomech 35: 873-880, 2002.

14. Li H, Li RX, Wan ZM, Xu C, Li JY, Hao QX, Guo Y, Liu L and Zhang XZ: Counter-effect of constrained dynamic loading on osteoporosis in ovariectomized mice. J Biomech 46: 1242-1247, 2013.

15. Nie J, Liu L, He F, Fu X, Han W and Zhang L: CKIP-1: A scaffold protein and potential therapeutic target integrating multiple signaling pathways and physiological functions. Ageing Res Rev 12: 276-281. 2013.

16. Lu K, Yin X, Weng T, Xi S, Li L, Xing G, Cheng X, Yang X, Zhang L and He F: Targeting WW domains linker of HECT-type ubiquitin ligase Smurf1 for activation by CKIP-1. Nat Cell Biol 10: 994-1002, 2008.

17. Baas D, Caussanel-Boude S, Guiraud A, Calhabeu F, Delaune-Henry E, Pilot F, Chopin E, Machuca-Gayet I, Vernay A, Bertrand S, et al: CKIP-1regulates mammalian and zebrafish myoblast fusion. J Cell Sci 125: 3790-3800, 2012.

18. Zhou ZC, Lei C, Liang K, Lei DL, Rui L and Yang XJ: CKIP-1 silencing promotes new bone formation in rat mandibular distraction osteogenesis. Oral Surg Oral Med Oral Pathol Oral Radiol 123: e1-e9, 2016.

19. Guo B, Zhang B, Zheng L, Tang T, Liu J, Wu H, Yang Z, Peng S, He X, Zhang $\mathrm{H}$, et al: Therapeutic RNA interference targeting CKIP-1 with a cross-species sequence to stimulate bone formation. Bone 59: 76-88. 2014.

20. Zhang L, Zhou Q, Song W, Wu K, Zhang Y and Zhao Y: Dual-functionalized graphene oxide based siRNA delivery system for implant surface biomodification with enhanced osteogenesis. ACS Appl Mater Interfaces 9: 34722-34735, 2017.

21. NASA: Strong bones and fewer renal stones for astronauts. International Space Station Program Science Office, NASA's Johnson Space Center. https://www.nasa.gov/mission_pages/ station/research/news/Strong_Bones.html. Accessed February 24, 2012

22. Wang QS, Zhang XC, Li RX, Sun JG, Su WH, Guo Y, Li H and Zhang XZ: A comparative study of mechanical strain, icariin and combination stimulations on improving osteoinductive potential via NF-kappaB activation in osteoblast-like cells. Biomed Eng Online 21: 46, 2015.

23. Wang QS, Wang GF, Lu YR, Cui YL, Li H, Li RX, Zhang XZ, Zhang CQ and Liu TJ: The combination of icariin and constrained dynamic loading stimulation attenuates bone loss in ovariectomy-induced osteoporotic mice. J Orthop Res 36: 1415-1424, 2018.

24. Tatsumi S, Ishii K, Amizuka N, Li M, Kobayashi T, Kohno K, Ito $\mathrm{M}$, Takeshita $\mathrm{S}$ and Ikeda K: Targeted ablation of osteocytes induces osteoporosis with defective mechanotransduction. Cell Metab 5: 464-475, 2007.

25. Wang Y, Liu W, Masuyama R, Fukuyama R, Ito M, Zhang Q, Komori H, Murakami T, Moriishi T, Miyazaki T, et al: Pyruvate dehydrogenase kinase 4 induces bone loss at unloading by promoting osteoclastogenesis. Bone 50: 409-419, 2012.

26. Livak KJ and Schmittgen TD: Analysis of relative gene expression data using real-time quantitative PCR and the 2(-Delta Delta C (T)) method. Methods 25: 402-408, 2001.

27. Allen DL, Bandstra ER, Harrison BC, Thorng S, Stodiec LS, Kostenui PJ, Moro S, Lacey DL, Hammond TG, Leinwand LL, et al: Effects of spaceflight on murine skeletal muscle gene expression. J Appl Physiol (1985) 106: 582-595, 2009.

28. Hatton DC, Yue Q, Dierickx J, Roullet C, Otsuka K, Watanabe M, Coste S, Roullet JB, Phanouvang T, Orwoll E, et al: Calcium metabolism and cardiovascular function after spaceflight. J Appl Physiol (1985) 92: 3-12, 2002. 
29. Crucian B and Sams C: Immune system dysregulation during spaceflight: Clinical risk for exploration-class missions. J Leukoc Biol 86: 1017-10118, 2009.

30. Zhang X, Wang Q, Wan Z, Li J, Liu L and Zhang X: CKIP-1 knockout offsets osteoporosis induced by simulated microgravity. Prog Biophys Mol Biol 122: 140-148, 2016.

31. Liu L, Guo Y, Chen X, Li R, Li Z, Wang L, Wan Z, Li J, Hao Q, $\mathrm{Li} \mathrm{H}$ and Zhang $\mathrm{X}$ : Three-dimensional dynamic culture of pre-osteoblasts seeded in HA-CS/Col/nHAP composite scaffolds and treated with alpha-ZAL. Acta Biochim Biophys Sin (Shanghai) 44: 669-677, 2012.

32. Guo Y, Zhang CQ, Zeng QC, Li RX, Liu L, Hao QX, Shi CH, Zhang XZ and Yan YX: Mechanical strain promotes osteoblast ECM formation and improves its osteoinductive potential. Biomed Eng Online 11: 1, 2012.

33. Liu Q, Guo Y, Wang Y, Zou X and Yan Z: miR-985p promotes osteoblast differentiation in MC3T3E1 cells by targeting CKIP-1. Mol Med Rep 17: 4797-4802, 2018.

34. Barger-Lux MJ and Recker RR: Bone microstructure in osteoporosis: Transilial biopsy and histomorphometry. Top Magn Reson Imaging 13: 297-305, 2002.

35. Sturmer EK, Seidlova-Wuttke D, Sehmisch S, Rack T, Wille J, Frosch KH, Wuttke W and Stürmer KM: Standardized bending and breaking test for the normal and osteoporotic metaphyseal tibias of the rat: Effect of estradiol, testosterone and raloxifene. J Bone Miner Res 21: 89-96, 2006.

36. Li J, Yang S, Li X, Liu D, Wang Z, Guo J, Tan N, Gao Z, Zhao X, Zhang J, et al: Role of endoplasmic reticulum stress in disuse osteoporosis. Bone 97: 2-14, 2017

37. Sun LW, Luan HQ, Huang YF, Wang Y and Fan YB: Effects of local vibration on bone loss in -tail-suspended rats. Int J Sports Med 35: 615-624, 2014.

38. Wang RN, Green J, Wang Z, Deng Y, Qiao M, Peabody M, Zhang Q, Ye J, Yan Z, Denduluri S, et al: Bone morphogeneticprotein (BMP) signaling in development and human diseases. Genes Dis 1: 87-105, 2014

39. Nohe A, Keating E, Knaus P and Petersen NO: Signal transductionof bone morphogenetic protein receptors. Cell Signal 16 291-299, 2004

40. Cicciù M: Real opportunity for the present and a forward step for the future of bone tissue engineering. J Craniofac Surg 28: 592-593, 2017.

41. Herford AS and Cicciù M: Recombinant human bone morphogenetic protein type 2 jaw reconstruction in patients affected by giant cell tumor. J Craniofac Surg 21: 1970-1975, 2010.

42. Herford AS, Cicciù M, Eftimie LF, Miller M, Signorino F and Famà F: Rhbmp-2 applied as support of distraction osteogenesis: A split-mouth histological study over nonhuman primates mandibles. Int J Clin Exp Med 9, 17187-17194, 2016.

43. NakashimaK,Zhou X,Kunkel G,ZhangZ,DengJM,BehringerRR and Crombrugghe B: The novel zincfinger-containing transcription factor osterix is required forosteoblast differentiation and bone formation. Cell 108: 17-29, 2002.
44. Simonet WS, Lacey DL, Dunstan CR, Kelley M, Chang MS, Lüthy R, Nguyen HQ, Wooden S, Bennett L, Boone T, et al: Osteoprotegerin: A novel secreted protein involved in theregulation of bone density. Cell 89: 309-319, 1997.

45. Yasuda H, Shima N, Nakagawa N, Mochizuki SI, Yano K, Fujise N, SatoY GotoM, Yamaguchi K, Kuriyama M, et al: Identity of osteoclastogenesis inhibitory factor(OCIF) and osteoprotegerin $(\mathrm{OPG})$ : A mechanism by which $\mathrm{OPG} / \mathrm{OCIF}$ inhibits osteoclastogenesis in vitro. Endocrinology 139: 1329-1337, 1998.

46. Ferrari-Lacraz S and Ferrari S: Do RANKL inhibitors (denosumab) affect inflammationand immunity? OsteoporosInt 22: 435-446, 2011.

47. Kuhn MC, Willenberg HS, Schott M, Papewalis C, Stumpf U, Flohé S, Scherbaum WA and Schinner S: Adipocytesecreted factors increase osteoblast proliferation and theOPG/RANKL ratio to influence osteoclast formation. Mol Cell Endocrinol 349: 180-188, 2012.

48. Wang L, Zhang XZ, Guo Y, Chen XZ, Li RX, Liu L, Shi CH, Guo $\mathrm{C}$ and Zhang $\mathrm{Y}$ : Involvement of BMPs/Smad signaling pathway in mechanical response in osteoblasts. Cell Physiol Biochem 26: 1093-1102, 2010.

49. Olsten ME, Weber JE and Litchfield DW: CK2 interacting proteins: Emerging paradigms for CK2 regulation? Mol Cell Biochem 274: 115-124, 2005.

50. Song DH, Dominguez I, Mizuno J, Kaut M, Mohr SC and Seldin DC: CK2 phosphorylation of the armadillo repeatregion of beta-catenin potentiates Wnt signaling. J Biol Chem 278: 24018-24025, 2003.

51. Jansen JH, Eijken M, Jahr H, Chiba H, Verhaar JA, van Leeuwen JP and Weinans H: Stretch-induced inhibited of Wnt/bete-catenin signaling in mineralizing osteoblasts. J Orthop Res 28: 390-396, 2010

52. Yu HC, Wu TC, Chen MR, Liu SW, Chen JH and Lin KM: Mechanical stretching induces osteoprotegerin in differentiating C2C12 precursor cells through noncanonical Wnt pathways. J Bone Miner Res 25: 1128-1137, 2010.

53. Barry PL: Good vibrations: a new treatment study by NASA-funded doctors could reverse bone loss experienced by astronauts in space. https://science.nasa.gov/science-news/ science-at-nasa/2001/ast02nov 1/. Accessed November 2, 2001.

54. Goodship AE, Cunningham JL, Oganov V, Darling J, Miles AW and Owen GW: Bone loss during long term space flight is prevented by the application of a short term impulsive mechanical stimulus. Acta Astronaut 43: 65-75, 1998

55. Zhang G, Guo B, Wu H, Tang T, Zhang BT, Zheng L, He Y, Yang Z, Pan X, Chow $\mathrm{H}$, et al: A delivery system targeting bone formation surfaces to facilitate RNAi-based anabolic therapy. Nat Med 18: 307-314, 2012. 